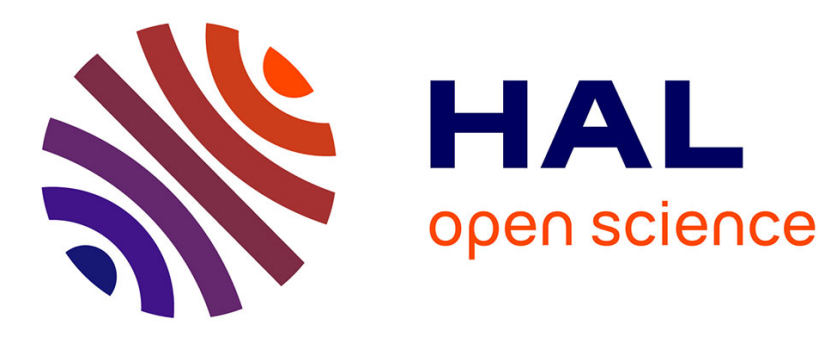

\title{
An Adaptive Cartography of DTV Programs
}

\author{
Jean-Gabriel Ganascia, Charles Madeira, Karan Fouladi
}

\section{To cite this version:}

Jean-Gabriel Ganascia, Charles Madeira, Karan Fouladi. An Adaptive Cartography of DTV Programs. EuroITV 2008 - 6th European Conference on Changing Television Environments, Jul 2008, Salzburg, Austria. pp.253-262, 10.1007/978-3-540-69478-6_34 . hal-00644915

\section{HAL Id: hal-00644915 https://hal.science/hal-00644915}

Submitted on 25 Nov 2011

HAL is a multi-disciplinary open access archive for the deposit and dissemination of scientific research documents, whether they are published or not. The documents may come from teaching and research institutions in France or abroad, or from public or private research centers.
L'archive ouverte pluridisciplinaire HAL, est destinée au dépôt et à la diffusion de documents scientifiques de niveau recherche, publiés ou non, émanant des établissements d'enseignement et de recherche français ou étrangers, des laboratoires publics ou privés. 


\title{
An Adaptive Cartography of DTV Programs
}

\author{
Jean-Gabriel Ganascia, Charles Madeira, and Karan Fouladi \\ Université Pierre et Marie Curie (Paris 6) \\ Laboratoire d'Informatique de Paris 6 (LIP6) \\ 104, avenue du Président Kennedy, F-75016, Paris, France \\ \{Jean-Gabriel.Ganascia, Charles.Madeira, Karan.Fouladi\} @lip6.fr
}

\begin{abstract}
With the development of information technologies and more precisely, the so-called digital convergence, the number of digital TV (DTV) programs available on any kind of DTV receiver (satellite, cable, terrestrial or IP) has increased dramatically. This has made the task of selecting a DTV program very complicated and time consuming. This paper proposes an intelligent graphical user interface (GUI) tool that gives a new appearance to the on-air and recorded DTV contents in order to enable DTV viewers to, quickly and in a user-friendly manner, select a program. The GUI tool is based on a synthetic cartography of DTV programs in which the shape generated reflects the DTV viewers' preferences over a period of time. These preferences are obtained by using any method for program clustering and recommendation. Results are demonstrated on the task of recommending programs in the context of the French terrestrial DTV by using a content-based approach.
\end{abstract}

Keywords: Intelligent Graphical User Interface, Digital TV Recommendation System.

\section{Introduction}

With the development of information processing, Internet, digital TV (DTV) and telecommunications, the so-called digital convergence becomes a reality 10. As a consequence, DTV viewers face the difficulty of quickly finding programs that best fits their preferences.

In order to aid DTV viewers for selecting programs more easily, several TV recommender systems have been proposed and developed in the last years. These systems take advantage of different approaches such as content-based filtering 116], collaborative filtering [214] and hybrid filtering 4312] for creating personalized Electronic Program Guides (EPG).

Nevertheless, even if these recommender systems restrict a large number of possibilities of choice, many of them remain. So, the graphical user interface (GUI) appearance used for presenting the personal EPG seems to be also a key point for the success of the DTV viewer choice. So far, the GUIs implemented on DTV receivers are usually awkward. Some of them only provide a textual list of DTV programs, while others are built on small views of current programs.

This paper constitutes an attempt to simplify visual access to DTV programs by using a novel GUI inspired by the technique of "Memory Islands" 6]. This

M. Tscheligi, M. Obrist, and A. Lugmayr (Eds.): EuroITV 2008, LNCS 5066, pp. 253-262, 2008.

(C) Springer-Verlag Berlin Heidelberg 2008 
novel GUI is based on a dynamic 2D cartography that represents personalized EPGs by clusters of DTV programs. As we shall see, personalization of EPGs can be generated by any existing recommender system approach.

In the next section, we recall what Memory Islands are, how they can be used to describe DTV programs and what the ontology on which program clustering is based on. Following this, we present the process for building Memory Islands in the context of the French terrestrial DTV programs, we briefly describe a content-based recommender system that we have developed to demonstrate Memory Islands, and we illustrate it with examples of some of the generated shapes. Finally, we conclude the paper and discuss ongoing work.

\section{Representing Personal EPG with Memory Islands}

\subsection{Memory Islands}

Memory Islands [6] were initially designed to ease browsing in e-books and more generally in digital textual documents. The original idea consists in transforming the linear structure of a textual document into a 2D space that maps its logical structure. It is inspired by the ancient "Arts of Memory" which relate to us how our memory capacity can be considerably increased by placing things into "locis" belonging to architectures that were previously learned [15. In other words, to "embody" the virtual content of e-books, Memory Islands anchor it on an automatically generated territory. In a way, it represents on a 2D space a digital content that appears linear at first sight. This representation corresponds to an increase of dimensions, which is quite unusual in information visualization, since the general aim is to reduce data dimensions 13. Note that the main goal here is not to focus attention on a particular item, but to represent a wide variety of contents and to stimulate human memories with an easy to remember picture, which facilitates user interactions with the contents.

The adopted solution consists in mapping each unit of virtual content onto a small deserted island, since uninhabited islands are known by sailors who simply sail around it, and therefore only know and name the coast (1D space) without worrying about the surface. These fictitious territories are designed to strike imagination and to remain anchored in our memory. Consequently, they must be distinguished the ones from the others as much as possible in order to reflect the structure of the digital content and to ease navigation. Therefore, the map generation program endeavors to produce forms that are as diversified as possible in order to build a realistic map of a territory that offers an overview of the content. This cartography takes advantage of historical studies of old maps by using legends, texts, colors, and more amazingly for us, relevant icons [8].

As presented in [6], Memory Islands may also represent any cultural/multimedia contents such as digital audio, digital video or DTV programs. In this case, the "islands" map the multimedia contents by some clustering. For example, clustering can be made with respect to the media theme, e.g. movies, news, etc. This allows to 
generate a weighted ordered tree that is transformed into a shape enriched by colors and icons correlated to each theme.

\subsection{Shape Generation Algorithm}

From a practical point of view, the logical structure of books corresponds to their table of contents. It may be transformed into a weighted ordered tree, of which node weights are computed according to the proportion of the book covered by the corresponding part. For instance, if a book containing 300 pages is divided in three parts containing respectively 50 pages, 100 pages and 150 pages, then the weights are respectively $1 / 6,1 / 3$ and $1 / 2$ for the parts 1,2 and 3 . This allows to generate a $2 \mathrm{D}$ shape from the weighted ordered tree.

More generally, any e-document structure can be assimilated to an ordered weighed tree, i.e. to an ordered tree of which nodes are associated to numbers corresponding to the weight of the corresponding parts. For instance, the number of characters for texts or the required space or time for some digital multimedia contents such as audio or video. A shape is generated from this tree.

The algorithm for generating shapes considers each weighted node of the structure of the e-document, i.e. each set of contents, as a sector of a circular disk, i.e. an angle associated with a radius. The angle corresponds to the proportion of the section in the whole book while the radius is computed taking into account the size of the section and its level. Moreover, some blank contents are virtually considered to separate blocs, which cut out the coast of the artificially generated island.

The algorithm takes as input an ordered labeled tree derived from the multimedia content organization. For instance, the content organization may cover five meta-themes - Society and Politics, Information, Culture, Youth and Fiction - while each one covers from two to four themes. It corresponds to the following multimedia organization that is voluntarily oversimplified, for the sake of the presentation: [[Society and politics, [Religion, Talk show, Society, War]], [Information, [News, Newsine]], [Culture, [History, Music, Theater]], [Youth, [Cartoon, Manga, Movies, Games]], [Fiction, [Thriller, Spies, Fantastic, SF]]].

Once this multimedia content organization ontology is given, the programs (e.g. next hour, next day or next week programs) and coefficients weighting their importance (e.g. program duration combined with its interest), are added to each leave of the tree. It gives a weighted ordered tree. More precisely, each node $\mathrm{N}$ of the weighted tree corresponds to a level of a hierarchy characterized by:

- A label (called label $(N))$, that is the characteristic associated to the level of the hierarchy. In the case of DTV programs, we restrict it to meta-themes and themes;

- A weight, which is the size of content covered by the nodes, e.g. a combination of the running time and preferences of DTV viewers;

- The depth of the node, i.e. the level of the node. For example, it may be 0 for the root of the hierarchy, 1 for meta-themes, 2 for themes and 3 for programs. 
Moreover, other parameters are involved in order to accentuate singularities of the generated island through their variations. Since our goal is to remember a form, we have to make each form as particular as possible. Here are all existing parameters:

- An internal size $\rho$. It may be predefined or computed as a function of the global running time or of the available space for digital audio and video.

- An eccentricity, $\epsilon$. It accentuates the length and the extension of capes, shaping them like islands.

- The number of blank contents between blocs. It is usually an input parameter, since it is difficult to get it by just reading the table of content. It helps to increase the size of gulfs in order to differentiate shapes. In the DTV program case, we use theme to build a separate section for each metadata.

The output of the algorithm generates a sequence of triplets $<\theta_{1}, \theta_{2}, \rho>$, each one corresponding to an arc in which the radius is $\rho$ on the sector $\left\langle\theta_{1}, \theta_{2}\right\rangle$. This sequence is automatically translated in Scalable Vector Graphics (SVG), generating a path passing by these generated points.

For instance, being given a set of programs described with the themes and the meta-themes given in the above described content organization, the previously referred algorithm generates the shape presented in figure 1.

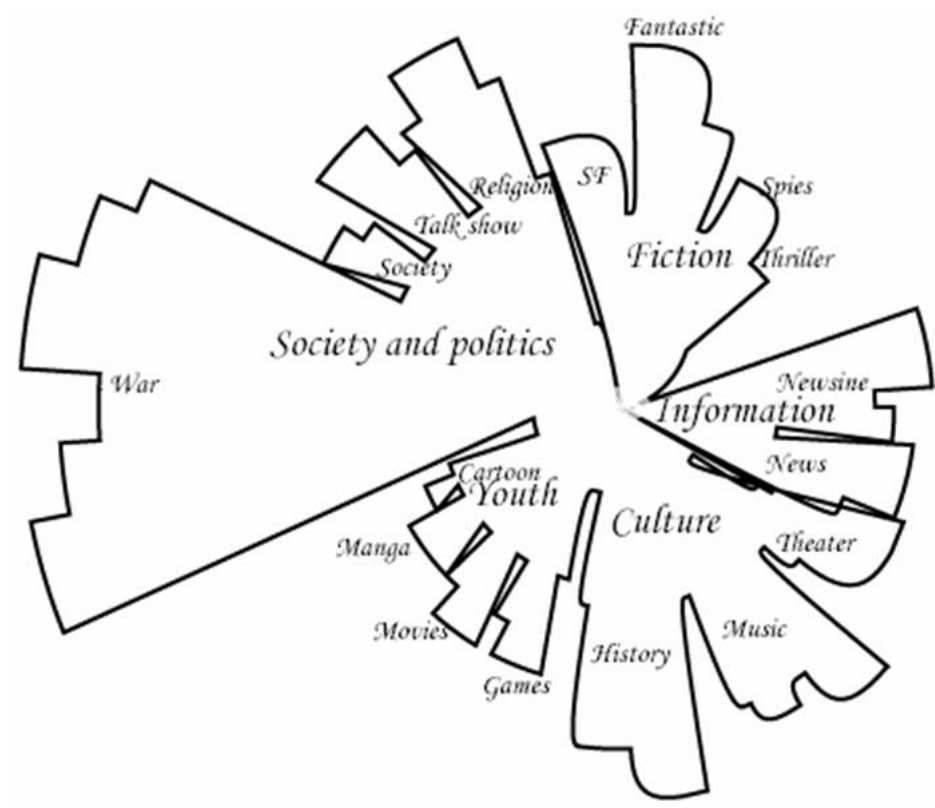

Fig. 1. A shape generated with a 5 meta-theme content organization 


\subsection{Ontology of Themes and Meta-themes}

As we have previously seen, the Memory Islands are built from a weighted ordered tree, which corresponds, in the case of cultural/multimedia contents, to the hierarchical organization of those contents. In the case of DTV programs, we identify the clusters related to a predefined organization. Three hierarchical levels are used to distinguish them:

- The first level is constituted by sub-themes. A group of homogeneous subthemes are assembled to build a theme.

- The second level corresponds to 39 classes that are characterized as themes, e.g. thriller, adventure, funny, drama, fantasy, cartoons, etc. They are built of the sub-themes groups. Each theme is associated with a meta-theme, which means that the ontology of theme consists in a hierarchy.

- The third level gives a broad definition of 12 classes as meta-themes, e.g. charm, fiction, society and politics, entertainment, youth, sport, sciences and nature, TV and shopping, information, do-it-yourself, culture, other.

Figure 2 contains themes associated with their corresponding meta-theme. Our first experiments using this setup demonstrate that it works very well in the case TV programs for at least two reasons. On the one hand, it can automatically determine themes and meta-themes from the metadata associated to each program. On the other hand, it corresponds to distinguishable sets of preferences associated to the social profile of families, which can facilitate a learning task.

\section{Applying Memory Islands to the French Terrestrial DTV}

In this section, we show a 2D cartography built from a set of DTV programs and automatically adapted to viewers' preferences. The first subsection explains the process of identifying themes and meta-themes for DTV Programs. The second subsection shows the building of a Memory Island from a DTV program set. The third subsection describes the process of adjusting DTV viewers' preferences by taking their behaviors into account. The fourth subsection shows how viewers' preferences shape the Memory Islands.

\subsection{Identifying Themes and Meta-themes of DTV Programs}

Once meta-themes and themes have been defined in the ontology introduced in section 2.3. we need to associate them with DTV programs. The goal consists in dynamically classifying EPG programs for a period of time in order to obtain the information required to build Memory Islands. This procedure is crucial for the usefulness of the Memory Islands generated in the context of the DTV.

Considering that program metadata obtained from the French Terrestrial DTV EPG are expressed in natural language, we propose an abstraction method that takes the metadata as input in order to identify their themes and meta-themes. 


$\begin{array}{llll}\text { Meta-themes } & \text { Themes } & \text { Meta-themes } & \text { Themes } \\ \text { charm } & \text { pornography } & \text { youth } & \text { youth } \\ \text { charm } & \text { eroticism } & \text { youth } & \text { cartoons } \\ \text { fiction } & \text { fiction } & \text { sport } & \text { sports } \\ \text { fiction } & \text { martial arts } & \text { sport } & \text { soccer } \\ \text { fiction } & \text { western } & \text { sport } & \text { motorsport } \\ \text { fiction } & \text { thriller } & \text { sport } & \text { swim sports } \\ \text { fiction } & \text { adventure } & \text { sciences\&nature } & \text { wildlife } \\ \text { fiction } & \text { funny } & \text { sciences\&nature sciences } \\ \text { fiction } & \text { drama } & \text { sciences\&nature hunting\&fishing } \\ \text { fiction } & \text { horror } & \text { tv\&shopping } & \text { tv shopping } \\ \text { fiction } & \text { science fiction } & \text { information } & \text { tv news } \\ \text { fiction } & \text { fantasy } & \text { do-it-yourself } & \text { do-it-yourself } \\ \text { society } & \text { society } & \text { do-it-yourself } & \text { culinary arts } \\ \text { society } & \text { politic } & \text { culture } & \text { culture } \\ \text { society } & \text { religion } & \text { culture } & \text { theater\&dance } \\ \text { society } & \text { VIP } & \text { culture } & \text { literature } \\ \text { society } & \text { history } & \text { culture } & \text { arts } \\ \text { entertainment } & \text { games\&gambling } & \text { other } & \text { diverses } \\ \text { entertainment } & \text { popular music } & \text { other } & \text { not announced } \\ \text { entertainment } & \text { show biz } & & \end{array}$

Fig. 2. List of 12 meta-themes and 39 themes defined in the ontology

This consists in simplifying the level of detail of the information available 7]. The input in here is composed of eight fields: Time, Channel, Title, Sub-title, Description, Category, Authors and Review. The procedure is performed as following:

- A parser uses a list of stop words to clean up the program metadata. This reduces each field to a bag of words;

- A list of keywords is selected by the TF-IDF (term frequency / inverse document frequency) information retrieval technique [11;

- The bag of words and the list of keywords are asserted into a knowledge-based system implemented with the NASA's C Language Integrated Production System (CLIPS V6-24) in order to identify themes and meta-themes of each program.

\subsection{Generating a DTV Program Memory Island}

Let us now suppose that we have a DTV program set composed of clustered programs. The first step required to generate a Memory Island consists in transforming this set into a weighted ordered tree. This is achieved by using the main theme identified by the clustering process, i.e. "Theme" attribute, which belongs to the theme ontology. The main theme associated with each program allows to cluster the program set into a three-level hierarchy. As previously said, the first level corresponds to the meta-theme, the second to the theme and the third to the programs themselves.

Therefore, we use the algorithm presented in section 2.2 to build the Memory Island corresponding to the weighted ordered tree. An example is given in Figure 3 . 


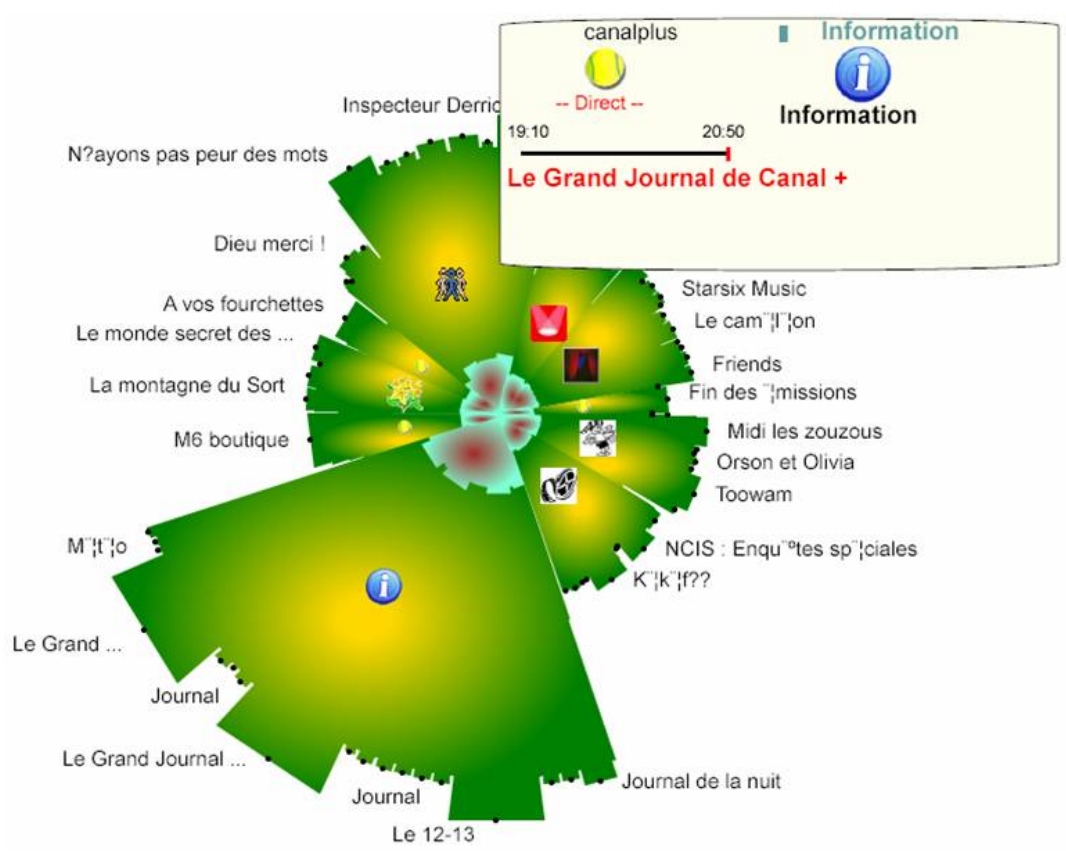

Fig. 3. A Memory Island with a pop-up for a focused program

We can see that the islands can be enriched with colors and icons corresponding to the most developed meta-themes and the main theme. Moreover, pop-up windows describing program characteristics can be automatically showed when a program is focused on. User interactions (selecting a theme, selecting a program, zooming a theme) are based on basic buttons of the remote control (up/down, left/right and +/-) that can be managed easily by anyone, without any requirement for learning.

\subsection{Adjusting DTV Viewers' Preferences}

In order to build personalized EPGs for testing the recommendation of programs by Memory Islands, we have been implementing a content-based recommender system that creates dynamic profiles reflecting viewers' behaviors.

Understanding Viewers' Behaviors. To understand how viewers behave, we interpret the events on the DTV receiver. First of all, we need to know which programs are available (on-air). This information is obtained by the EPG. Secondly, we need to monitor viewers' interactions with the DTV receiver. Viewers' interactions are described by a pair $\langle b, s\rangle$, where $b$ is a button on the remote control that is pressed at a time $t$ and $s$ is the current screen state on the receiver. The EPG and the viewers' interactions are then associated together, referring to the time scale matching. The matched information is then analyzed in order to learn the viewers' behaviors. 
This analysis is carried out in two stages: a syntax validation and a semantic validation. The syntax validation consists in combining interactions that operate on the collected data during a specified period of time. It generates an outline perspective on the viewers' interactions. This combination procedure is based on a set of interactions that lead to changing a channel. They are called main interactions. All programs watched on this specific channel are recorded in a devoted list. Other interactions such as volume increase or decrease are associated to this list as secondary interactions.

The semantic validation consists in evaluating the programs watched by the viewer at each period of time identified through syntax validation. The goal here is to rank the programs according to the time that the viewer spent on watching them and the reason of eventually changing the channel. In order to dynamically express the viewers' preferences, the program's rank contributes to reward or punish the associated abstract representation of the program introduced by the clustering method (see section [3.1). This plays a very important role in the performance of machine learning techniques [9].

Learning Viewers' Preferences. The abstract representation of programs is then matched to the viewers' profile in order to get their ranks. Using these ranks, a filtering process is carried out to arrange programs by decreasing order of relevance. Programs ranked higher occupy bigger regions on the generated Memory Island in order to reflect the viewers' preferences on screen. Machine learning techniques are advocated in this context to dynamically learn the viewers' preferences. The task consists in learning a function that ranks all programs according to user preferences. The goal of this task is to automatically update a pair $\langle r, w\rangle$, where $r$ is an abstract program representation and $w$ is a weight value associated with the abstract representation. We can use different learning strategies to recommend programs (short-term and/or long-term).
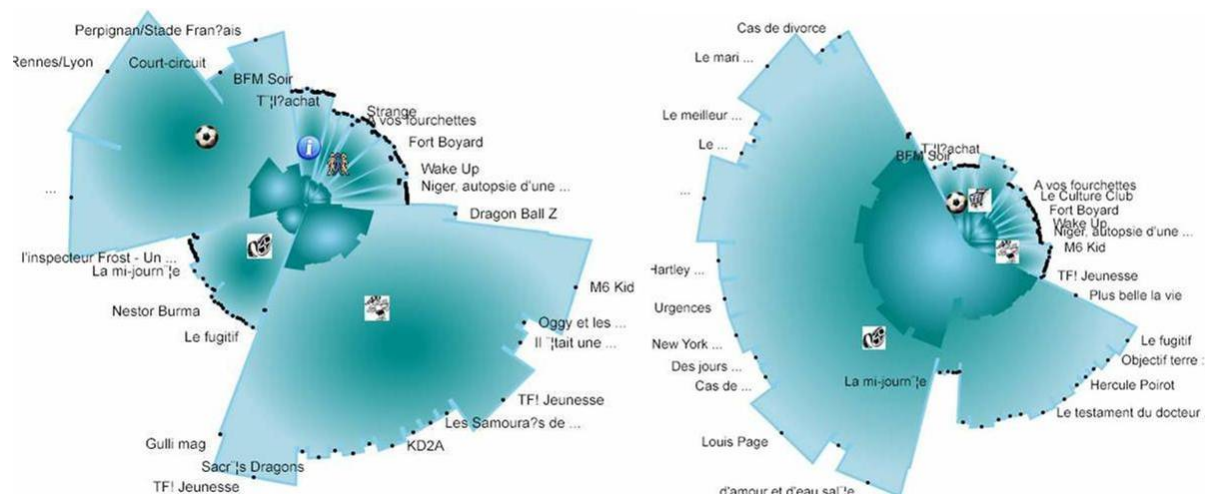

Fig. 4. Two Memory Islands showing the preferences for two kind of group of users 


\subsection{Adapting the Shape of Memory Islands through Viewers' Preferences}

In this section, we take into account a DTV program set of roughly two hundred programs and show them through two Memory Islands that reflect the preferences of two kind of group of users. The Memory Island presented on the left in Figure 4 corresponds to a group of users interested in watch football matches and youth programs. The Memory Island on the right corresponds to another group of users that is a film-loving.

\section{Conclusion and Future Work}

Intelligent GUIs for DTV receivers are a very interesting and original problem little investigated until now. Designing GUIs that facilitate the DTV viewer choice seems to be a key point for the next generation of DTV receivers. Moreover, they can significantly aid the recommendation of DTV programs made by automated systems.

This paper proposed a novel intelligent GUI tool that constitutes an alternative to the GUIs usually employed on DTV receivers. This novel GUI tool is based on a synthetic 2D cartography of DTV programs, inspired by the technique of Memory Islands, to enable DTV viewers to quickly select programs. It can be associated with any method for program clustering and recommendation in order to generate personalized EPGs.

The generation of shapes through Memory Islands has been illustrated for some personalized EPGs in the context of the French terrestrial DTV by using a content-based recommender system. The first results have demonstrated that desired programs can be easily located by using our system. In cooperation with a team of the IWEDIA Company and another of the LIMSI Laboratory, we are carrying out experiments with our system in the context of several groups of users. We will be evaluating, quantitatively and qualitatively, the behavior changes and the level of satisfaction of the different groups when using our system. More results will be available in the next months.

\section{References}

1. Ardissono, L., Gena, C., Torasso, P., Bellifemine, F., Chiarotto, A., Difino, A., Negro, B.: User Modeling and Recommendation Techniques for Personalized Electronic Program Guides. In: Ardissono, L., Kobsa, A., Maybury, M. (eds.) Personalized Digital Television: Targeting Programs to Individual Viewers, pp. 3-26. Kluwer, Dordrecht (2004)

2. Baudisch, P., Brueckner, L.: TV Scout: Guiding Users from Printed TV Program Guides to Personalized TV Recommendation. In: Proceedings of the 2nd Workshop on Personalization in Future TV, Malaga, Spain, pp. 157-166 (2002)

3. Buczak, A., Zimmerman, J., Kurapati, K.: Personalization: Improving Ease-ofUse, Trust and Accuracy of a TV Show Recommender. In: Proceedings of the 2nd Workshop on Personalization in Future TV, Malaga, Spain (2002) 
4. Hsu, S., Wen, M.-H., Lin, H.-C., Lee, C.-C., Lee, C.-H.: AIMED - A Personalized TV Recommendation System. In: Proceedings of the 5th European Interactive TV Conference, Amsterdam, The Netherlands, pp. 166-174 (2007)

5. Ganascia, J.-G.: RECIT: reprsentation cartographique et insulaire de texts. In: Proceedings of the CIFT 2004 (Colloque International sur la Fouille de Texte), La Rochelle, France (2004), http://archivesic.ccsd.cnrs.fr/sic_00001258/en/

6. Ganascia, J.-G.: AC3 - Automatic Cartography of Cultural Contents. In: Lévy, P.P., Le Grand, B., Poulet, F., Soto, M., Darago, L., Toubiana, L., Vibert, J.-F. (eds.) VIEW 2006. LNCS, vol. 4370, pp. 253-263. Springer, Heidelberg (2007)

7. Giunchiglia, F., Walsh, T.: A Theory of Abstraction. Artificial Intelligence 56(2-3), 323-390 (1992)

8. Jacob, C.: L'empire des cartes. Albin Michel (1992)

9. Mitchell, T.: Machine Learning. McGraw-Hill, Sing (1997)

10. O'Driscoll, G.: The essential guide to digital set-top-boxes and interactive DTV. Prentice Hall, Englewood Cliffs (1999)

11. Singhal, A.: Modern Information Retrieval: A Brief Overview. Bulletin of the IEEE Computer Society Technical Committee on Data Engineering 24(4), 35-43 (2001)

12. Tsunoda, T., Hoshino, M.: Automatic metadata expansion and indirect collaborative filtering for TV program recommendation system. Multimedia Tools and Applications 36(1-2), 37-54 (2008)

13. Tufte, E.: The Visual Display of Quantitative Information, 2nd edn. Graphics Press (2001)

14. Wang, J., Polwelse, J., Fokker, J., Reinders, M.: Personalization of a Peer-to-peer Television System. In: Proceedings of the 4th European Interactive TV Conference, Athens, Greece, pp. 147-155 (2006)

15. Yates, F.: The Art of Memory. Penguin Books (1969)

16. Zimmerman, J., Kurapati, K., Buczak, A., Schaffer, D., Gutta, S., Martino, J.: TV Personalization System. Design of a TV Show Recommender Engine and Interface. In: Ardissono, L., Kobsa, A., Maybury, M. (eds.) Personalized Digital Television: Targeting Programs to Individual Viewers, pp. 27-52. Kluwer, Dordrecht (2004) 